\title{
BMJ Open A survey of social support for exercise and its relationship to health behaviours and health status among endurance Nordic skiers
}

\author{
Paul J Anderson, ${ }^{1}$ Ralph S Bovard, ${ }^{2}$ Zhen Wang, ${ }^{3,4}$ Timothy J Beebe, ${ }^{3,4}$ \\ Mohammad Hassan Murad ${ }^{1}$
}

To cite: Anderson PJ, Bovard RS, Wang Z, et al. A survey of social support for exercise and its relationship to health behaviours and health status among endurance Nordic skiers. BMJ Open 2016;6:e010259. doi:10.1136/bmjopen-2015010259

- Prepublication history and additional material is available. To view please visit the journal (http://dx.doi.org/ 10.1136/bmjopen-2015010259)

Received 13 October 2015 Revised 28 April 2016 Accepted 29 April 2016

CrossMark

For numbered affiliations see end of article.

Correspondence to Dr Zhen Wang; wang.zhen@mayo.edu

\section{ABSTRACT}

Objectives: Regular exercise is a key component of obesity prevention and $48 \%$ of Americans do not meet minimum guidelines for weekly exercise. Social support has been shown to help individuals start and maintain exercise programmes. We evaluated social support among endurance athletes and explored the relationship between social support for exercise, health behaviours and health status.

Design: Survey.

Setting: The largest Nordic ski race in North America. Participants: 5433 past participants responded to an online questionnaire.

Outcome measures: Social support, health behaviours and health status.

Results: The mean overall support score was 32.1 $(S D=16.5$; possible range $=-16.0$ to 88.0 ). The most common forms of social support were verbal such as discussing exercise, invitations to exercise and celebrating the enjoyment of exercise. We found that an increase of 10 points in the social support score was associated with a $5 \mathrm{~min}$ increase in weekly selfreported exercise (5.02, 95\% Cl 3.63 to 6.41$)$.

Conclusions: Physical activity recommendations should incorporate the importance of participation in group activities, especially those connected to strong fitness cultures created by community and competitive events.

\section{INTRODUCTION}

More than two-thirds (69\%) of Americans are overweight or obese and there is irrefutable evidence that regular physical activity effectively prevents obesity and other chronic diseases such as cardiovascular disease, diabetes, cancer, hypertension, depression and osteoporosis. ${ }^{1}$ Yet $25 \%$ of Americans report no leisure time physical activity, less than half $(48 \%)$ report that they meet the 2008 physical activity guidelines of $>150 \mathrm{~min} /$ week, and fewer than $10 \%$ meet the physical activity guidelines when measured with actigraphy. $^{23}$

\section{Strengths and limitations of this study}

- The study characterised patterns of social support among endurance athletes who already participate in a strong fitness subculture.

- We analysed the strength of the relationship between social support for exercise, health behaviours and self-reported health status.

- Self-reporting of exercise is an important limitation.

In contrast, many citizen endurance athletes participate in fitness cultures that encourage physically active lifestyles associated with high self-reported health status, high prevalence of healthy lifestyle behaviours and remarkably low levels of obesity. ${ }^{4}$ Social Learning Theory suggests that such social support reinforces self-efficacy (the ability to effectively carry out planned actions) and helps individuals overcome a variety of difficult barriers to behaviour change. ${ }^{5}$ Sallis applies these concepts to nutrition and physical activity and has created a validated survey in order to analyse the balance between forms of encouragement and discouragement from family and friends. ${ }^{6}$ Self-efficacy and social support from families and friends may function synergistically to help people start new physical activity programmes, maintain their current regimens and improve their future performance. ${ }^{7}$ In the light of this, it is plausible that many endurance athletes achieve success through participation in fitness cultures that provide strong social support for their athletic endeavours. To the best of our knowledge, patterns of social support for exercise behaviours among Nordic skiers have not been characterised.

The purpose of this descriptive study was to characterise patterns of social support 
among endurance athletes who already participate in the fitness subculture supported by The American Birkebeiner Nordic ski race. This study also attempts to generate hypotheses about the relationship between social support for exercise, health behaviours and selfreported health status.

\section{METHODS}

\section{Study population}

The study population included individuals who had ever participated in at least one event sponsored by the American Birkebeiner Ski Foundation. The American Birkebeiner ski race (aka 'The Birkie') was founded in 1973 and it is now the largest Nordic ski race in North America attracting over 12000 participants in 2015. The American Birkebeiner Ski Foundation (ABSF) champions the race as a 'year round lifestyle choice for outdoor fitness enthusiasts of all levels'. In addition to Nordic ski races, Birkie events now include a fall mountain-bike marathon, spring and summer running races on the Birkebeiner trail, family centred ski weekends, skijoring events (skiing while pulled by a dog) and a fat-tire on-snow bicycle race. The hallmark Birkebeiner events are the $54 \mathrm{~km}$ classic ski race and the $50 \mathrm{~km}$ skate ski race, as well as the $25 \mathrm{~km}$ Korteloppet which may be completed in skate or classic technique. Each participant in a Birkie event receives a unique 'Birkie ID number' which was coupled with their email address to form an individual participant record.

\section{Data collection}

Between 30 October 2014 and 8 December 2014, 23611 Birkie participants received an email invitation to participate in an online survey. The online survey explored demographics, health status and health behaviours (see Supporting Information). Health status and health behaviour questions were modelled after the Behavioral Risk Factor Surveillance System (BRFSS) and questions commonly used in the National Health and Nutrition Examination Survey (NHANES). Health status questions explored how participants would rate their current health status, how they felt their health compared with others, and whether they had any chronic conditions relevant to sport participation. Health behaviour questions explored topics such as nutrition, exercise habits, sleep patterns, smoking history and alcohol use. Self-reported demographic and physiological indicators were collected in order to calculate body mass index (BMI). This study was approved by the Mayo Clinic Institutional Review Board (IRB).

\section{Social support score}

To calculate social support for exercise, we generally followed the validated method described by Sallis which arrives at a social support score by subtracting the values for negative social support questions from the values for positive social support responses. ${ }^{6}$ In this analysis, a support value was calculated for each of the 13 social support queries in question 10 (see Supporting Information) by assigning a numerical weight (0-4) to each response (eg, discussed exercise with me). We then added the total for family support and friend support to arrive at a support value for each query. For each social support query in question 10 , the total value could be between 0 and 8 . Further, to calculate the total social support score for an individual, we added together the support values from the 11 positive support queries and subtracted the values for the 2 negative support queries. Total social support scores for an individual in this study could range from -16 to 88 .

\section{Statistical analysis}

We conducted descriptive analyses to summarise the survey respondents' demographics, health status, health behaviours and social support for exercise. Simple and multiple logistic regression models and linear regression models were constructed to evaluate the association between health status, health behaviours and social support. We adjusted age, gender, BMI, marriage status (married vs not married), employment (employed vs unemployed/retired), education (college degrees or above vs no college degree) and income (household income $>\$ 100000$ vs $<=\$ 100000)$. Tests were deemed statistically significant if two tailed $p<0.05$. All statistical analyses were conducted using Stata V.13.1 (StataCorp, College Station, Texas, USA).

\section{RESULTS}

Survey responses were received by 5433 participants (response rate $=23 \%$ ) and were included in analysis. Overall, survey respondents reported an average age of 47.9 years ( $\mathrm{SD}=14.2$, Range: 13,88$)$ and there was a slight predominance of males $(64.5 \%)$ to females. The mean BMI was 23.8 ( $\mathrm{SD}=2.9$, Range: 15.1, 44.4) and the percentage of respondents who were obese was only $3.23 \%$. The majority of respondents were married $(71 \%)$, employed full-time $(66.42 \%)$, college educated or above $(86.2 \%)$, and slightly more than half had a household income greater than $\$ 100000$ (54.2\%). Most participants reported very good/excellent health status $(88.3 \%)$, that they were as fit or more fit than their peers $(99.1 \%)$, that they were free of physical limitations $(82.8 \%)$ and free from depression/low $\operatorname{mood}(93.1 \%)$.

Healthy behaviours were common in this group with only $14.4 \%$ eating vegetables less than one time daily, $18.8 \%$ eating fruit less than one time daily and only $5.84 \%$ reporting no leisure time physical activity. For other health status and health behaviour information, please see table 1 .

Four questions in the survey asked about motivation for exercise. Over $95 \%$ of survey respondents reported that they are highly motivated to exercise by the simple act of signing up for the Birkebeiner or similar events. Many Birkie skiers $(69 \%)$ are motivated to stay in shape by the 
thought that they are as fit or more fit than others in their same life situation (age, gender, etc). While exercising with others motivates Birkie skiers to sign up for races $(75 \%)$, most reported that they would still sign up for races even if their family or friends did not do so $(78 \%)$.

Table 1 Demographics and biometrics for respondents to the Birkie health survey, 2015

\begin{tabular}{|c|c|}
\hline & $\begin{array}{l}\text { Birkebeiner } \\
\text { skiers (SD) } \\
(n=5433)\end{array}$ \\
\hline \multicolumn{2}{|l|}{ Demographics and biometrics } \\
\hline Mean age & $\begin{array}{l}47.9(14.2) \\
\text { (range: 13, 88) }\end{array}$ \\
\hline Male gender & $64.5 \%$ \\
\hline Mean BMI & $\begin{array}{l}23.8(2.9) \\
\text { (range: } 15.1,44.4)\end{array}$ \\
\hline $\mathrm{BMI} \geq 30$ & $3.23 \%$ \\
\hline Married & $71.36 \%$ \\
\hline Employed full time (>35 h) & $66.42 \%$ \\
\hline $\begin{array}{l}\text { Four year college education or } \\
\text { more }\end{array}$ & $86.2 \%$ \\
\hline Household Income >\$100 000 & $54.2 \%$ \\
\hline \multicolumn{2}{|l|}{ Health status } \\
\hline $\begin{array}{l}\text { Very good/excellent health } \\
\text { status }\end{array}$ & $88.3 \%$ \\
\hline As fit/more fit than peers & $99.1 \%$ \\
\hline No physical limitations & $82.8 \%$ \\
\hline Free from depression/low mood & $93.1 \%$ \\
\hline \multicolumn{2}{|l|}{ Health behaviours } \\
\hline Sleep $<7$ h/night & $65.1 \%$ \\
\hline Snoring & $36.3 \%$ \\
\hline $\begin{array}{l}\text { Unintentionally falling asleep } \\
\text { during daytime }\end{array}$ & $31.3 \%$ \\
\hline Eating fruit less than 1 time daily & $18.2 \%$ \\
\hline $\begin{array}{l}\text { Eating vegetables less than } 1 \\
\text { time daily }\end{array}$ & $14.4 \%$ \\
\hline Current smokers & $3.37 \%$ \\
\hline Drink alcohol & $90.5 \%$ \\
\hline $\begin{array}{l}\text { Moderate alcohol consumption } \\
\text { (0-2 drinks per occasion) }\end{array}$ & $83.9 \%$ \\
\hline Binge drinking, ever & $10.35 \%$ \\
\hline No leisure time physical activity & $5.84 \%$ \\
\hline Arthritis & $18.7 \%$ \\
\hline
\end{tabular}

A slight majority of skiers reported that they most often train alone (53\%), but during an average week most $(70.85 \%)$ exercise with others at least once. The majority of skiers were introduced to Nordic skiing by a family member or a friend $(79 \%)$ and many $(35 \%)$ reported that they most often train with family or friends (table 2).

\section{Social support scores}

Overall, the mean social support score for all participants was $32.3(\mathrm{SD}=16.5$; possible range $=-16.0$ to 88.0$)$. For the various queries about types of social support, the most prominent form of social support was discussing exercise (mean=4.6, $\mathrm{SD}=2.2$; possible range $=0.0-8.0$ ). Other prominent forms of social support included exercising together (mean=4.1, $\mathrm{SD}=1.9$ ), offers to exercise together (mean=3.9, $\mathrm{SD}=2.0$ ), talking about how much exercise is enjoyed (mean=3.7, $\mathrm{SD}=2.3$ ), planning for exercise on recreational outings (mean $=3.5, \mathrm{SD}=2.2$ ) and planning activities around the need for exercise (mean=3.0, $\mathrm{SD}=2.2$ ). Negative social interactions such as complaints about exercising (mean=0.7, $\mathrm{SD}=1.1$ ) and criticism for exercising (mean $=0.5, \mathrm{SD}=1.0$ ) received very low scores. For the relationship between the various queries about types of social support, please see figure 1 .

\section{The relation of social support to health status and health behaviours}

Individuals with high levels of social support were more likely to report very good/excellent health status $(\mathrm{OR}=1.31$; CI 1.21 to $1.41 ; \mathrm{p}<0.001)$ and less likely to report problems with depression/low $\operatorname{mood}(\mathrm{OR}=0.84$, CI 0.77 to $0.92 ; \mathrm{p}<0.001)$. Each 10 point increase in the social support score was associated with a $5 \mathrm{~min}$ increase in weekly exercise time, and those reporting higher levels of social support were more likely to exercise greater than $150 \mathrm{~min}$ each week $(\mathrm{OR}=1.13$, CI 1.08 to $1.18 ; \mathrm{p}<0.001)$. Those reporting higher levels of social support were less likely to report less than once daily consumption of fruit $(\mathrm{OR}=0.88$, CI 0.83 to 0.93 ; $\mathrm{p}<0.001)$ or vegetables $(\mathrm{OR}=0.78$, CI 0.74 to 0.84 ; $\mathrm{p}<0.001)$. Increased social support scores were not significantly associated with tobacco or alcohol use or reports of no leisure time activity (table 3 ).

Table 2 Motivation and training patterns among American Birkebeiner participants

\begin{tabular}{ll}
\hline Introduced to Nordic Skiing by a family member or friend & Agree/strongly agree (\%) \\
I MOST OFTEN train with a family member or friend & 79.26 \\
I MOST OFTEN train alone & 35.13 \\
I am highly motivated to exercise by signing up for races like the Birkie & 53.71 \\
If my friends and family did not sign up for races like the Birkie, I would NOT participate & 95.85 \\
Exercising with friends motivates me to sign up for races like the Birkie & 21.88 \\
ON AVERAGE, I meet with other people to exercise at least 1 time per week & 75.31 \\
Motivated to stay in shape by the thought that I am staying more fit than others in my life situation & 70.85 \\
\hline
\end{tabular}




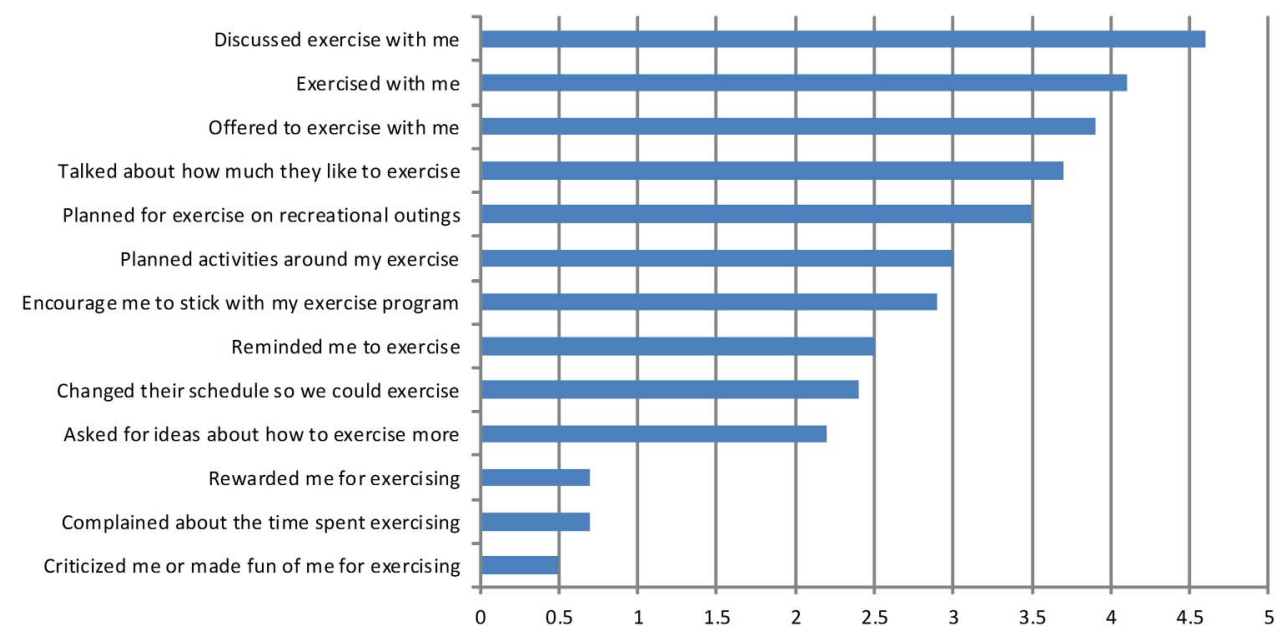

Figure 1 Mean score for each social support query among American Birkebeiner participants.

\section{DISCUSSION}

While there are a few studies of motivation among professional Nordic athletes, ours study appears to be the first to provide a general profile of health and social support behaviours in a population of citizen Nordic skiers. ${ }^{8}{ }^{9}$ Among Birkie skiers, the most common forms of social support were predominantly verbal such as discussing exercise, talking about the enjoyment of exercise and making offers to exercise with someone else. Exercising together, and making or changing plans around exercise were also very strong forms of social support. Social learning theory would suggest that social support through verbal processing helps skiers overcome barriers that involve motivation, the technical aspects of training and racing, and helps transmit 'cultural' elements of Nordic skiing such as trail etiquette, personal resilience and lifestyle behaviours important to better performance.

The behaviour of adjusting plans in order to exercise with others and exercising together may address even more barriers to training. While much social support was reported as verbal, Birkie skiers also reported significant support from regular group exercise. While a slight majority of respondents train alone most of the time $(53 \%)$, most report exercise with others at least once weekly $(71 \%)$. Further, respondents would still sign up for races without friend or family support, but do gain some motivation to race from training with others. While these responses seem difficult to reconcile, it may be that on the one hand the predominance of solitary training coupled with the determination to sign up for races regardless of social support suggests a high level of self-efficacy. On the other hand, the pattern of weekly exercise with others and the fact that skiers do sign up for races because of motivation derived from others suggests that any pre-existing selfefficacy may be reinforced by regular doses of exercisespecific social support.

Exercise-specific social support has been found to increase exercise adherence more than generalised

Table 3 Association between social support scores and health status, health behaviour

\begin{tabular}{|c|c|c|c|c|}
\hline Health status & $\begin{array}{l}\text { Unadjusted OR } \\
(95 \% \mathrm{Cl})\end{array}$ & p Value & $\begin{array}{l}\text { Adjusted OR } \\
(95 \% \mathrm{Cl})^{\star}\end{array}$ & p Value \\
\hline Very good/excellent health status & 1.26 (1.18 to 1.35$)$ & $<0.001$ & $1.31(1.21$ to 1.41$)$ & $<0.001$ \\
\hline $\begin{array}{l}\text { Feel depression/low mood } \\
\text { Health behaviour }\end{array}$ & $0.87(0.80$ to 0.94$)$ & $<0.001$ & 0.84 (0.77 to 0.92$)$ & $<0.001$ \\
\hline More than 150 min exercise & 1.14 (1.09 to 1.19$)$ & $<0.001$ & 1.13 (1.08 to 1.18$)$ & $<0.001$ \\
\hline Exercise time $(\mathrm{min}) \dagger$ & 5.63 (4.35 to 6.93$)$ & $<0.001$ & 5.02 (3.63 to 6.41$)$ & $<0.001$ \\
\hline No leisure time physical activity & $0.76(0.49$ to 1.16$)$ & 0.20 & $.68(0.36$ to 1.29$)$ & 0.24 \\
\hline Sleep $>7$ h/night & $1.10(1.06$ to 1.15$)$ & $<0.001$ & 1.07 (1.02 to 1.12$)$ & 0.004 \\
\hline Eating fruit less than 1 time daily & 0.85 (0.81 to 0.90$)$ & $<0.001$ & $0.88(0.83$ to 0.93$)$ & $<0.001$ \\
\hline Eating vegetables less than 1 time daily & $.74(0.70$ to 0.79$)$ & $<0.001$ & $0.78(0.74$ to 0.84$)$ & $<0.001$ \\
\hline Current smokers & $1.00(0.77$ to 1.30$)$ & 0.98 & $1.14(0.86$ to 1.52$)$ & 0.37 \\
\hline Drink alcohol & $1.00(0.94$ to 1.07$)$ & 1.00 & $1.00(0.93$ to 1.08$)$ & 0.93 \\
\hline Moderate alcohol consumption (0-2 drinks per occasion) & 1.07 (1.01 to 1.13$)$ & 0.03 & 1.03 (0.96 to 1.09$)$ & 0.42 \\
\hline Binge drinking, ever & $0.94(0.87$ to 1.01$)$ & 0.08 & $0.98(0.91$ to 1.06$)$ & 0.62 \\
\hline
\end{tabular}

${ }^{*}$ Adjusted for age, gender, BMI, marriage status (married vs not married), employment (employed vs unemployed/retired), education (college degrees or above vs no college degree) and income (household income $>\$ 100000$ vs $<=\$ 100000$ ). BMl, body mass index. †Reported as mean changes, not OR. 
social support and family and friends are particularly influential in this process. ${ }^{10}{ }^{11}$ The majority of respondents in our study (79\%) lend support to this idea since they report that they were specifically introduced to Nordic skiing by a family member or friend. Further, some of the most common social support behaviours in our survey involved planning for exercise on recreational outings and scheduling activities around exercise, both of which presume strong exercise-specific social support from friends and family.

In addition to social support patterns, our study explored the relationship between social support, health status, health behaviours and exercise patterns. Studies of runners in the USA and Switzerland have previously documented elevated health status and increased healthy behaviours in these populations. ${ }^{4}{ }^{12}$ Our survey revealed many of the same patterns of elevated health status in skiers, and also revealed a positive relationship between overall social support and weekly minutes of exercise. In general, for every $5 \mathrm{~min}$ increase in weekly minutes of exercise reported, there was a 10 point increase in the self-reported social support score. In addition, reporting of depression/low mood was less likely to report strong social support $(\mathrm{OR}=0.84, \mathrm{p}<0.001)$. These findings point to a positive link between physical activity levels and social connection and underscore the importance of physical activity promotion efforts that emphasise group activities, especially participation in strong fitness cultures.

\section{Limitations}

Self-reporting of exercise is an important limitation in our study and some of our questions on motivation have not been validated. Actigraphy studies show that people report far more physical activity than they actually perform when measured with an accelerometer. The positive relationship between self-reported weekly minutes of exercise and social support score is valuable for hypothesis generation, but in future studies it would be valuable to give participants an activity-monitoring device and a social support survey to see if there is a relationship between social support and monitoring data. In addition, we did not collect physiological data or examine medical records from participants and this limits our ability to characterise the actual health status of our participants. Future studies could involve simple physical examination, chart reviews and basic physiology testing for a subset of participants. Dietary recall is also difficult and this may have caused an overestimation of fruit and vegetable intake in this group. Future studies could use a formal $24 \mathrm{~h}$ dietary recall form, a weekly food diary or an online programme. ${ }^{3}$ The relatively low response rate of $23 \%$ might raise concerns about the precision of our study, potential nonresponse bias and the overall inferential value of our study findings. However, recent evidence suggests that a high response rate may not be the best predictor of overall survey quality. ${ }^{13}{ }^{14}$ Nonetheless, generalisations from our study findings should be made with caution. Finally, the survey was internet-based and some individuals who do not use the internet regularly may not have received or completed the study. This may have excluded some older skiers or those without internet service.

\section{Conclusions}

Patterns of social support for exercise in Endurance Nordic skiers reveal the importance of verbal processing and regular group exercise for the initiation and maintenance of a physically active lifestyle. While endurance athletes appear to have high levels of self-efficacy, they also appear to draw on social support to bolster their motivation and skills and overcome barriers to better performance, especially support from family and friends. These insights drawn from exercise-specific social support in Nordic skiers are valuable for clinicians and public health practitioners attempting to promote increased physical activity. Those wanting to initiate, maintain or increase physical activity regimens should receive guidance that emphasises the importance of physical activities in groups, especially family of friend groups connected to larger fitness cultures that often surround community races or large national or international competitive events.

\section{Author affiliations}

${ }^{1}$ Division of Preventive, Occupational, and Aerospace Medicine, Mayo Clinic, Rochester, Minnesota, USA

${ }^{2}$ Department of Occupational and Environmental Medicine Residency, Health Partners, Saint Louis Park, Minnesota, USA

${ }^{3}$ Robert D. and Patricia E. Kern Center for the Science of Health Care Delivery, Rochester, Minnesota, USA

${ }^{4}$ Division of Health Care Policy and Research, Division of Health Sciences

Research, Mayo Clinic, Rochester, Minnesota, USA

Contributors PJA, RSB and MHM contributed to the study design. PJA, TJB and MHM developed the survey. ZW analysed the results. PJA wrote the initial draft of the manuscript. PJA, RSB, ZW, TJB and MHM revised the manuscript.

Funding This research received no specific grant from any funding agency in the public, commercial or not-for-profit sectors.

Competing interests None declared.

Ethics approval The study was approved by the Mayo Clinic Institutional Review Board (IRB \#: 14-003594) prior to implementation.

Provenance and peer review Not commissioned; externally peer reviewed.

Data sharing statement No additional data are available.

Open Access This is an Open Access article distributed in accordance with the Creative Commons Attribution Non Commercial (CC BY-NC 4.0) license, which permits others to distribute, remix, adapt, build upon this work noncommercially, and license their derivative works on different terms, provided the original work is properly cited and the use is non-commercial. See: http:// creativecommons.org/licenses/by-nc/4.0/

\section{REFERENCES}

1. Warburton DE, Nicol CW, Bredin SS. Health benefits of physical activity: the evidence. CMAJ 2006;174:801-9.

2. Prevention CfDCa. State Indicator Report on Physical Activity, 2010 US Department of Health and Human Services, 2010.

3. Tucker JM, Welk GJ, Beyler NK. Physical activity in US: adults compliance with the Physical Activity Guidelines for Americans Am J Prev Med 2011;40:454-61. 
4. Williams PT. Relationship of distance run per week to coronary heart disease risk factors in 8283 Male runners. The National Runners' Health Study. Arch Inter Med 1997;157:191-8.

5. Bandura A. Social learning theory. Englewood Cliffs, NJ: Prentice Hall, 1977.

6. Sallis JF, Grossman RM, Pinski RB, et al. The development of scales to measure social support for diet and exercise behaviors. Prev Med 1987;16:825-36.

7. Duncan TE, McAuley E. Social support and efficacy cognitions in exercise adherence: a latent growth curve analysis. J Behav Med 1993;16:199-218.

8. Lemyre PN, Hall HK, Roberts GC. A social cognitive approach to burnout in elite athletes. Scand J Med Sci Sports 2008;18:221-34.

9. Pensgaard AM, Roberts GC. Elite athletes' experiences of the motivational climate: the coach matters. Scand J Med Sci Sports 2002;12:54-9.
10. Oka RK, King AC, Young DR. Sources of social support as predictors of exercise adherence in women and men ages 50 to 65 years. Womens Health 1995;1:161-75.

11. Wallace LS, Buckworth J, Kirby TE, et al. Characteristics of exercise behavior among college students: application of social cognitive theory to predicting stage of change. Prev Med 2000;31: 494-505.

12. Chatton A, Kayser B. Self-reported health, physical activity and socio-economic status of middle-aged and elderly participants to a popular road running race in Switzerland: better off than the general population? Swiss Med Wkly 2013;143:w13710.

13. Johnson TP, Wislar JS. Response rates and nonresponse errors in surveys. JAMA 2012;307:1805-6.

14. Davern M. Nonresponse rates are a problematic indicator of nonresponse bias in survey research. Health Serv Res 2013;48:905-12. 